\title{
Experiencia del \\ desplazamiento: roles de \\ género en mujeres de Cali'
}

Valentina Riaño Lara² Daniela Suárez Valbuena³ valenriano@javerianacali.edu.co_dsuarez97@javerianacali.edu.co

Diana Marcela Villarreal Yudy Alejandra Zambrano Cuero $^{4} \quad$ Ojeda $^{5}$

dianavillarreal@javerianacali.edu.co yudyzambrano@javerianacali.edu.co

Artículo corto de investigación recibido el 21/02/2018

y aprobado el 11/03/2018

Cómo citar este artículo:

Suárez Valbuena, D., Villareal Cuero, D., Riaño Lara, V., \& Zambrano Ojeda,Y.(2018). Experienciadel desplazamiento: roles de género en mujeres de Cali. Trans-Pasando Fronteras, (11). 


\section{Resumen}

El desplazamiento forzado es una de las causas del conflicto armado interno, implica toda migración por despojo o huida de territorios con alta frecuencia de enfrentamientos entre el estado y grupos armado ilegales, y tiene grandes consecuencias sobre la vida de las personas tales como, pérdidas económicas, del capital social, entre otras (Ibañez y Velásquez, 2008). Sobre dichas pérdidas Reynaga (como se cita en Venturoli, 2009) expone que, implican una reorganización de los roles asignados a hombres y mujeres. En Colombia, el número de desplazados supera los seis millones, y hay un mayor número de mujeres que de hombres (Registro único de víctimas, 2017).

Por ésta razón, se plantea la presente investigación cualitativa, desde un diseño fenomenológico, entendido como el método para acceder a las representaciones sobre una situación, desde la vivencia de cada persona (Salgado, 2007), teniendo como objetivo el conocer si la experiencia del desplazamiento por conflicto armado, influye en los comportamientos relacionados con los roles de género de tres mujeres entre los 20 y 60 años residentes de la ciudad de Cali, mediante el uso de una entrevista semi-estructurada. En los resultados se evidenció que después del desplazamiento, las pérdidas generadas llevaron a estas mujeres a tener comportamientos socialmente atribuidos a los hombres en mayor

\footnotetext{
${ }^{1}$ Las autoras como parte de un ejercicio de clase de Investigación cualitativa, realizaron el presente artículo, Experiencia del desplazamiento: roles de género en mujeres de Cali. Del cual se han derivado reflexiones por parte de las autoras, presentadas en la Catedra internacional Ignacio Martin Baró en la universidad Javeriana y en el III encuentro nacional de estudiantes de psicología.

²Estudiante de séptimo semestre de la carrera de Psicología en la Pontificia Universidad Javeriana ${ }^{3}$ Estudiante de séptimo semestre de la carrera de Psicología en la Pontificia Universidad Javeriana ${ }^{4}$ Estudiante de séptimo semestre de la carrera de Psicología en la Pontificia Universidad Javeriana ${ }^{5}$ Estudiante de séptimo semestre de la carrera de Psicología en la Pontificia Universidad Javeriana
} 
frecuencia después de la migración, para suplir la falta y lograr responder a las exigencias de su nuevo contexto, conservando en su mayoría funciones culturalmente otorgadas a las mujeres como la preparación de alimentos y el cuidado de los hijos; implicando con esto, una reorganización en el rol de género; Por lo cual se concluye que dichos cambios comportamentales si tienen relación con la situación de desplazamiento vivenciada.

Palabras clave: Rol de género; Mujeres; Consecuencias del conflicto; Desplazamiento.

\section{Introducción}

Colombia es un país que ha experimentado una larga guerra durante más de 50 años, en los cuales se ha observado un gran número de muertes y víctimas del conflicto armado interno, ello se evidencia en los resultados ofrecidos por el informe de la Agencia de la ONU para los refugiados (ACNUR) de 1985 a 2015, en donde se delata que en Colombia hay un total de 6.9 millones de desplazados, convirtiéndolo en el país con mayor número de desplazados internos del mundo, superando las cifras registradas en Siria con 6.6 millones, e Irak con 4.4 millones (ACNUR, 2015).

En concordancia con lo anterior, el Registro Único de Víctimas (RUV) del 1 de febrero del 2017, expone que de 8.048.252 de víctimas del conflicto armado, 7.108.181 han sido desplazados internos, donde la cifra de las mujeres (3.627.350) supera la de los hombres (3.444.552) (Unidad de Víctimas, 2017). Estos datos son una muestra del impacto que ha tenido el conflicto armado en el país, y la necesidad de generar investigaciones al respecto. 
Ahora bien, para entender éstas cifras es imprescindible comprender el conflicto armado en Colombia, por ésta razón, a continuación se presentará una breve contextualización de lo que ha sido la historia del conflicto hasta el día de hoy.

Para comenzar, no se sabe con exactitud cuáles son las causas del conflicto armado, pues son muchas y diversas, sin embargo, lo que ha hecho de este conflicto algo particularmente extenso, fueron una serie de factores definidos por la Comisión Histórica del Conflicto (2015), tales como: el narcotráfico y la economía de guerra, el secuestro y la extorsión, la precariedad institucional, el paramilitarismo, las armas y urnas, el fracaso de la reforma agraria, el sistema político clientelista/localista, y el círculo vicioso de la violencia, los cuales significaron un fortalecimiento por parte de los entes involucrados.

Durante los más de 50 años que duró la guerra, y mientras más fuertes se hacían los combatientes, las modalidades de guerra tomaron diferentes formas, de manera que las personas víctimas del conflicto sufrieron las consecuencias de la guerra a través de factores como: el secuestro, la extorsión, el reclutamiento ilícito de niños, niñas y adolescentes, la tortura, el homicidio en persona protegida, los asesinatos selectivos y masacres, las amenazas, los delitos contra la libertad y la integridad social, las minas antipersonas, las municiones sin explotar, los artefactos explosivos no convencionales, los ataques y pérdidas de bienes civiles, los atentados contra bienes públicos, el despojo de tierras o el desplazamiento.

Todas estos hechos, estuvieron presente en la mayoría de los sectores del país, y dada la cantidad de personas víctimas, se decidió, bajo el liderazgo del gobierno nacional colombiano, dar fin 
a ésta larga guerra, estableciendo un acuerdo de paz que los incluyera a todos y que estuviera conforme a los intereses de las partes involucradas. Es por eso que ya el año 2012 se dio inicio a los diálogos para el acuerdo de paz entre las Farc y el gobierno del país, y durante 4 largos años se realizaron diferentes modificaciones, acordes a las inquietudes de ambos entes, de tal manera que en 2016 se firma el acuerdo final para la terminación del conflicto y la construcción de una paz estable y duradera, el cual desde enero del 2017 comenzó a tomar medidas para el cumplimiento del mismo.

Lo planteado no quiere decir que hayan dejado de existir problemáticas internas en términos de violencia, pero han reducido, tal y como lo demuestra el Centro de Recursos para el Análisis de Conflictos (CERAC, 2015), pues gracias a los acuerdos se han evitado más de 1.500 muertes causadas por el conflicto, y las cifras delatan que hay 473 menos heridos de la guerra.

A pesar de lo anterior, todavía quedan heridas de este conflicto, y si de verdad se quiere establecer una paz estable y duradera habrá que fijarse en las consecuencias del mismo, para promover la reparación y la justicia a las víctimas.

Dada la cantidad de consecuencias del conflicto, ésta investigación quiere centrarse en las experiencias de los desplazados, pues son ellos los que quedaron vivos después de haber sufrido la guerra, y son ellos quienes tienen la verdad para contarle al país lo realmente sucedió, para hacer lo posible por la no repetición. Así pues, si se quiere trabajar con los desplazados, se hace necesario profundizar en qué se entiende por desplazado y cuáles fueron sus consecuencias sobre la vida de las personas. 
A partir de lo anterior, según la ley 1448 de 2011, una víctima del desplazamiento forzado es definida como:

Aquellas personas que individual o colectivamente hayan sufrido un daño por hechos ocurridos a partir del $1^{\circ}$ de enero de 1985, como consecuencia de infracciones al Derecho Internacional Humanitario o de violaciones graves y manifiestas a las normas internacionales de Derechos Humanos, ocurridas con ocasión del conflicto armado interno (ley 144, 2011, art. 3)

Los estudios realizados por Ibañez y Velásquez (2008), encontraron que los principales efectos del desplazamiento en las personas son: pérdidas de activos, pérdidas de capital social, la dificultad para entrar en el mercado laboral y las mejoras en el ámbito educativo. El primero hace referencia a las pérdidas de recursos económicos tales como: mercados financieros formales o informales, el capital físico, tierras y viviendas, donde el impacto económico puede llegar a ser más fuerte cuando la persona receptora del ingreso en la familia deja de estar presente, ya sea por asesinato, secuestro o muerte natural. Cabe resaltar que el 61\% de los hogares desplazados reciben sus ingresos gracias al trabajo del hombre, mientras que el 39\% por la mujer.

Además de las pérdidas de activos, hay una dificultad para entrar en el mercado laboral porque las habilidades de las personas que han sido desplazadas no son buscadas en el sector urbano, siendo difícil recuperar el estatus económico, lo que hace a las víctimas del desplazamiento más proclives a la pobreza (Ibañez y Velásquez, 2008). 
En cuanto a las pérdidas del capital social, después del desplazamiento hay una destrucción de redes sociales, lo cual podría significar mayor dificultad para salir adelante en su nueva residencia, porque de alguna manera los familiares y amigos brindan apoyo económico y social en momentos de crisis. No obstante, aunque el desplazamiento puede generar consecuencias negativas, se encuentra una mejoría en el ámbito educativo, pues las ciudades brindan mayor oferta de educación que las que se encuentran en el campo (Ibañez y Velásquez, 2008).

Sumado a lo anterior, el desplazamiento tuvo otro tipo de consecuencias particularmente interesantes, pues tal y como lo expresa Reynaga (como se cita en Venturoli, 2009), las familias que se trasladaron del campo a la ciudad experimentaron un proceso de reorganización de los roles asignados a los hombres y a las mujeres. Sin embargo, la manera en que se da ésta reorganización, es decir, los cambios cualitativos que se presentan desde antes de ser víctimas del conflicto, hasta el momento en que ingresan a la nueva ciudad y se adaptan a ella, no ha sido muy estudiada, es por ésto que la presente investigación busca dar respuesta a ¿Cómo la experiencia del desplazamiento por conflicto armado, puede influye en los comportamientos relacionados con los roles de género de tres mujeres entre los 20 y 60 años residentes de la ciudad de Cali? Pero para responder a lo anterior, se hace necesario explicar qué se va a entender en ésta investigación por género, cuáles son los roles de género atribuidos a las mujeres y a los hombres en el campo.

Así pues, para comenzar el género hace referencia al conjunto de identificaciones, atributos, comportamientos y funciones socialmente construidos al hombre y a la mujer, conteniendo el significado social y cultural que se asignan a esas diferencias biológicas 
(Alcántara, 2013). En esta medida, la sociedad establece roles estereotipados para ambos sexos, donde al hombre se le confieren aquellas actividades que tienen mayor valor económico, social, político y sociocultural; mientras que a la mujer se le delega aquellas labores relacionadas con lo doméstico, las tareas reproductivas y de crianza, es decir, con el carácter mismo de ser madre (Gandulfo, s.f).

En concordancia con lo anterior, Andrade, Alvis, Jiménez, Redondo y Rodríguez (2016) plantean que el rol de la mujer en Colombia, particularmente en el marco del conflicto se caracteriza porque ella tiende a ser obligada a servir y se clasifica como pertenencia de otro, lo que aumenta la probabilidad de que se construya la idea de sujeto con poca autonomía, alterando su autoconcepto (percepción sobre sí mismo) y afectando su salud psicológica y corporal. En la misma línea de ideas, Soto (2014) considera que la percepción de la mujer en este contexto está marcado por un pensamiento deshumanizado y patriarcal. Así pues, la mujer tiende a presentar un alto grado de vulnerabilidad sexual, material y psicosomática. La primera se da al asignársele una imagen corporal como arma de guerra y limitada a la satisfacción de las necesidades del hombre. La segunda es porque generalmente no cuentan con un empleo formal, mientras que la tercera se debe a que son blanco recurrente de actos terroristas y reclutamientos.

Además, en una de las investigaciones de éste autor, que tenía como objetivo evocar experiencias de ocho mujeres en situación de desplazamiento antes, durante y después del conflicto vivenciado, por medio de una entrevista con enfoque biográfico y narrativo, concluyó que existe una diferencia en la naturaleza de las interacciones por parte de las mujeres en contexto de guerra y posteriormente desde su condición de desplazadas, resaltando 
su papel activo y central en la construcción de memoria individual y la memoria colectiva (Soto, 2014). Ahora bien, se sabe que los roles asignados a los hombres y las mujeres cambian dependiendo del contexto en el que se desarrollen, por lo tanto, si las personas que han sido desplazadas eran principalmente campesinas, se ve necesario hacer una distinción entre los roles que atribuían a los hombres y a las mujeres en el campo, y cómo estos fueron cambiando al llegar a la ciudad.

En este sentido, Álvarez, Díaz y Saiz (como se cita en Güiza, Rodríguez-Barajas, Ríos, y Moreno, 2016) plantean que "las mujeres rurales han sido objeto de una histórica discriminación y desigualdad global, pues las estructuras patriarcales han sido propias de las relaciones sociales en el ámbito campesino" (p.119). Además, resaltan el hecho que ellas se encuentran en una situación de vulnerabilidad y desigualdad en oportunidades de formación y desarrollo, pues se las limita a las actividades relacionadas con la familia y el hogar. Con respecto al rol de la mujer y el hombre en el campo, Reynaga (como se cita en Venturoli, 2009) destaca tres actividades que se realizan en el campo: actividades agrícolas, artesanales y domésticas, realizando la diferenciación del rol a partir de ellas. Por un lado, en las actividades agrícolas, el hombre se encarga de: el arado, barbecho, chacmeo (volteado) de tierras, aporque, riego, control de plagas, y el traslado de la cosecha; mientras las mujeres se encargan de la selección, preparación y recolección de las semillas en el surco, así como del deshierbe. También ella es la que decide que parte de la cosecha será destinada para el consumo de la casa y la venta.

Las actividades artesanales realizadas por las mujeres se centran en la elaboración de: mantas, cinturones y tejidos en telar de cintura, mientras que los hombres crean ponchos, frazadas y bay- 
etas en telar de cintura o de pedal. Finalmente, en lo referente a las actividades domésticas, son realizadas principalmente por las mujeres, encargadas de: cocinar, cuidar a los hijos y el hogar, conservar y lavar la ropa, moler granos, trasladar agua, entre otras.

Referente a los hombres desplazados, buscaban trabajo como ayudantes de albañilería, comerciantes de ganado, carpintería o mecánica, mientras que las mujeres como empleadas domésticas, comerciantes ambulatorias (principalmente de frutas y verduras), o feriantes (Reynaga, como se cita en Venturoli, 2009).

Finalmente, el estudio realizado por Romero-Acosta y Contreras (2015), que tuvo como objetivo plantear una propuesta de intervención, a partir de una revisión teórica exhaustiva alrededor de las reflexiones sobre el rol de la mujer y el desplazamiento forzado, plantea como conclusiones que no hay estudios sobre el papel de la mujer en el post-conflicto ni tampoco del territorio colombiano, por esta razón se hace necesario investigar más sobre el tema y diseñar programas de intervención orientadas al empoderamiento de la mujer.

Teniendo en cuenta lo escrito a lo largo del trabajo, se puede decir que existen muchas investigaciones que hablan sobre el rol de género en mujeres víctimas del conflicto armado, sin embargo, ninguna de ellas analiza los cambios antes y después de ser desplazadas.

Por ésta razón la presente investigación tuvo como objetivo general, conocer si la experiencia del desplazamiento por conflicto armado influye en los comportamientos relacionados con el rol de género de tres mujeres entre los 20 y 60 años residentes de la ciudad de Cali. Para cumplirlo, se plantearon como objetivos 
específicos: entender los significados sobre la experiencia de desplazamiento por conflicto armado, identificar las conductas relacionadas con el rol de género antes y después de este acontecimiento, y reconocer si el desplazamiento por el conflicto armado influye en las acciones relacionadas con el rol de género.

\section{Método}

\section{Tipo de investigación}

De acuerdo a Salgado (2007), esta investigación fue de tipo cualitativa, ya que tuvo como objetivo conocer la realidad subjetiva desde la experiencia de las participantes, sus significados, percepciones y representaciones, además, buscó entender cómo se han construido sus tramas de acción y de sentido alrededor del rol de género y el desplazamiento por conflicto armado.

Por otro lado, en lo referente a los diseños de la investigación cualitativa descritos, la presente fue de tipo fenomenológico, puesto que, permitió abordar los significados de la experiencia individual; partiendo del punto de vista de cada una de las participantes, su contexto relacional, historia de vida, corporalidad y narración. En esta medida, este tipo de diseño facilitó el logro de los objetivos de la investigación, en tanto que permitió una mejor comprensión de las representaciones del rol de género y el significado de las experiencias que perciben las participantes, al estar inmersas en un contexto violento que las obliga a desplazarse de su lugar de origen y porque resalta la importancia de la interpretación del investigador alrededor de este fenómeno (Salgado, 2007). 


\section{Participantes}

Los participantes en esta investigación fueron, tres mujeres desplazadas entre los 24 y 56 años de edad, donde los criterios de inclusión considerados fueron: tener entre 20 y 60 años, ser mujer y haber sido desplazada a Cali por causa del conflicto armado. En tanto a los criterios de exclusión, se plantearon los siguientes: no ser mujer, ser menor de edad y haber sido desplazada por causas diferentes al conflicto armado interno.

En lo referente a la técnica de muestreo empleada, fue por bola de nieve, ya que facilitó la aproximación a las realidades culturales de poblaciones con difícil acceso, así que se contactó con un informante clave que permitió la vinculación de los individuos al objetivo de la investigación (Quintana, 2016). En este caso, se solicitó la colaboración de una trabajadora independiente en el Oeste de Cali, quien ofreció el contacto de dos vecinas y una de ellas, posibilita la conexión con la última participante.

\section{Técnica de recolección de información}

La técnica empleada para la recolección de información fue la entrevista semiestructurada, la cual comprendió una guía de preguntas donde se encontraban establecidos los tópicos que se debían abordar, para asegurar la recolección de datos suficientes para la investigación, teniendo en cuenta lo expuesto por Bonilla y Rodríguez (1997) respecto al orden y contenido que favorece la obtención de información válida durante el encuentro. Así pues, las investigadoras diseñaron la entrevista organizada en tres grandes apartados, correspondientes al "Inicio", "Desarrollo" y "Cierre". El primero, cuenta con siete puntos, dirigidos a establ- 
ecer el rapport o clima de la entrevista, comunicar a las entrevistadas sobre el objetivo de la investigación y realizar preguntas demográficas, buscando indagar sobre las características personales de las entrevistadas (Bonilla y Rodríguez, 1997).

En el apartado de "Desarrollo", se establecieron dos categorías de análisis previas: "Rol de género" y "Consecuencias del conflicto armado", las cuales abarcaron los ejes temáticos propios de la investigación, relacionados con los objetivos y la revisión del estado del arte. En este sentido, para la categoría de "Rol de género" se fijaron las siguientes subcategorías: función doméstica y ocupación, mientras que en la de "Consecuencias del conflicto armado" se establecieron: pérdidas de capital social, pérdidas económicas, razones de migración, sentimientos relacionados al lugar de residencia, valoración de respuesta por parte de las autoridades y retos como mujer en un contexto de conflicto.

Así pues, dicho apartado contó con diez preguntas en el eje temático de "Rol de género" y cuatro preguntas en el de "Consecuencias del conflicto armado". En este sentido y de acuerdo con Bonilla y Rodríguez (1997), las preguntas fueron abiertas y de tipo experiencial, de opiniones y sentimientos. El primer tipo de preguntas indagaron sobre lo que hacen o han hecho las participantes en relación con el fenómeno, las preguntas de opiniones, pretendían conocer lo que las entrevistadas piensan sobre los temas asociados al objetivo de la investigación. Finalmente, las referente a los sentimientos, estaban encaminadas a conocer cuáles fueron y son las respuestas emocionales de las tres mujeres frente al fenómeno. Para finalizar, el apartado de "Cierre" pretendía dar por finalizada la entrevista, haciendo una síntesis del desarrollo de esta y agradeciendo a las participantes. 


\section{Procedimiento}

Para la primera fase se contactó con una informante clave, a quien se le comentó el objetivo de la investigación y facilitó el encuentro con las dos participantes que cumplían con los criterios de inclusión y una de ellas, posibilita el contacto con la última participante. De esta manera se seleccionaron las participantes y se contactó con ellas, a quienes se les presentaron el objetivo de la investigación y se las invitó a participar, una vez que accedieron se acordó el día, la hora y el lugar de la entrevista.

En la segunda fase, luego de la firma del consentimiento informado, se realizó una entrevista a cada participante, siguiendo la guía de entrevista y haciendo uso de una grabadora de voz. Por otro lado, en la tercera fase se realizó la transcripción de las entrevistas, las cuales se organizaron y categorizaron para el mejor manejo de la información.

Para finalizar, la cuarta fase consistió en la redacción de los resultados, para elaborar su análisis y discusión, teniendo en cuenta la revisión del estado del arte. Cabe resaltar que por confidencialidad de las personas entrevistadas responderán a nombres ficticios, donde "Luz Mary" es una mujer de 36 años, "Teresa" de 56 y "Juana" de 24 años de edad.

\section{Análisis de datos}

Se realizó un análisis temático de los resultados, ya que permitió identificar, organizar y reportar datos ricos en detalle, para la adecuada comprensión del objeto de estudio relacionado con los tópicos teóricos (Braun y Clarke, como se cita en Miles, Tonon 
y Alvarado, 2012). De esta manera, transcribieron las entrevistas, posteriormente se realizó una primera lectura que posibilitó identificar los códigos previamente establecidos. Continuando con la selección de los fragmentos pertenecientes a las categorías y subcategorías ya mencionadas en apartados anteriores, concluyendo con la interpretación relacionada de estas.

\section{Consideraciones éticas}

El presente trabajo de investigación tuvo en cuenta las consideraciones éticas basadas en la ley 1090 de 2006 del Ministerio para la Protección Social de Colombia, específicamente el artículo 2, apartado número 5, la cual expone la confidencialidad de datos que debe ejercer el psicólogo colombiano sea cual sea su labor (Ministerio para la Protección Social, 2006).

Es por ello que se dejó constancia a partir del consentimiento informado, que la entrevista sería aplicada por estudiantes de quinto semestre de la Pontificia Universidad Javeriana Cali, quienes estarían en continua supervisión de su profesora de Investigación en Psicología III, durante el proceso de diseño de la investigación y recolección de datos. Se aseguró el carácter de confiabilidad en las respuestas de los participantes, para fines académicos. Del mismo modo, las respuestas obtenidas a partir de las entrevistas tendrían un carácter privado, guardando la identidad de cada uno de los participantes (Ministerio para la Protección Social, 2006). 


\section{Resultados}

A continuación se presentan los resultados, los cuales se encuentran relacionados con los objetivos y las categorías de análisis establecidas para la investigación.

\section{Rol de género}

De acuerdo con el rol de género, las funciones domésticas, referidas a todas aquellas actividades realizadas en el hogar y de crianza, las participantes verbalizaron que antes de ser desplazadas ellas:

"Cocinaba en la casa de mi suegra (...) y hacía el oficio (...) tenía una niña y estaba ahí en la casa no más, con ella" (Luz Mary, 36 años). También, una mujer menciona que "Vivía en una vereda de San Carlos en putumayo, allá trabajaba y vivía en una finca (...) a veces a las 11 de la noche llegaba la guerrilla yo tenía que cocinarles" (Teresa, 56 años)

Durante el proceso de desplazamiento, hasta su llegada a Cali, ellas manifestaron que:

"Ellos (sus hijos) solitos se van, solo me levanto a hacerles desayuno (...) los despacho y se van, luego cojo mi maleta y me voy a trabajar, por ahí a las 5 o 5 y media voy llegando, ahí a hacer comida. Los niños llegan por ahí a la 1 y yo les dejo haciendo almuerzo y ya" (Luz Mary, 36 años). Además, otra participante comenta que "No hacía nada más, nadie me daba trabajo, y hacia las cosas de la casa y "(Actualmente) llegó a las 4 de la tarde y tengo que llegar derechito a cocinar y oficio (...) las cosas de la casa, para eso es la mamá" (Teresa, 56 años). 
Por otro lado, en cuanto a la subcategoría de ocupación, las participantes verbalizaron que antes de ser desplazadas ellas:

"Vivía en una vereda de san Carlos en putumayo, allá trabajaba y vivía en una finca" (Teresa, 56 años), otra verbalizó: "Yo estudiaba, yo terminé el bachiller allá" (Juana, 24 años). También, se afirma que "ahí cocinaba en la casa de mi suegra... y hacia el oficio" (Luz Mary, 36 años).

Sin embargo, después del desplazamiento, afirman que:

"Aquí, trabajo limpiado máquinas para embutidos" (Luz Mary, 36 años). Así mismo, una de las entrevistas menciona que "yo tenía gallinas en cantidad, más de 200 gallinas y tocó salir al parque a venderlas baratísimas porque nos sacaron de allá... de eso nos manteníamos" y "acá a Cali Llegué en el 2010, acá trabajo en una casa de familia" (Teresa, años). Por su parte, una mujer comenta "Ahora estoy estudiando, ahora estoy aquí en el instituto estudiando farmacología"(Juana, 24 años)

\section{Consecuencias del conflicto armado}

Entre las consecuencias del conflicto se encuentran las pérdidas de capital social, donde las participantes verbalizan que:

"En el putumayo mataron al papá de mi hija, después que lo mataron a él me dio muy duro" (Luz Mary, 36). Otra participante menciona "Pensando en que pueda tener mis cositas y ayudar a mi familia, en algún momento traérmelos a ellos para acá para que puedan vivir mejor, tranquilos como nosotros y no tener que pasar por todo eso. Además nunca puedo verlos, y es difícil hablar con ellos."(Juana, 24). 
De igual manera, en relación con las pérdidas económicas, declararon que:

"Estuvimos ahí (en putumayo) como 3 años, no teníamos ni casa ni nada, de eso nos manteníamos (...) no hacía nada más, nadie me daba trabajo, y hacia las cosas de la casa." (Teresa, 56 años). Mientras que una de las mujeres expresa "Para nosotros fue muy difícil porque acá no teníamos conocidos ni nada y empezamos de cero" (Juana, 24).

En otro orden de ideas, para razones de migración expresaron lo siguiente:

"Llegaron los paracos, y todos nos vinimos de por allá, por ejemplo mi suegra y toda su familia, ahí yo me junté con el papá de mis hijos y él me trajo para acá, por trabajo" (Luz Mary, 36). Otra participante añade "Nos sacaron y di la declaración en pasto, la guerrilla nos sacó, dijo que nos largáramos (...) si nos hubiésemos quedado ahí nos hubiesen matado, porque buscaba a mi patrón y nos salimos (...) Yo convencí a mi familia que nos viniéramos porque allá no había nada, nada. Ahora si esta bueno. Porque pensé que acá había mejor vida, el colegio, ahora ya está bueno allá (...) A veces íbamos caminando y comenzaban a echar plomo" (Teresa, 56).

Además, una de las entrevistadas dice que "De Cali todo el mundo decía que Cali era lo mejor que habían muchas más oportunidades que acá se vivía tranquilo, en paz, por eso nos vinimos"(Juana, 24). 
Así mismo, para sentimientos relacionados al lugar de migración, respondieron:

"Cuando yo vivía allá (en putumayo) vivía bien, me sentía bien, cómoda (...) En mercaderes, no me gustaba porque era muy aburrido (...) En pasto no me gustaba vivir y por eso me fui, aquí (en Cali) sí, me siento bien." (Luz Mary, 36). Por otro lado, una participante expresa "Mira que yo recuerdo que en el pueblo sentía mucha rabia, rabia con la gente porque nadie lo ayudaba a uno (...) no me gustaría vivir allá, porque uno pasó por muchas cosas y el miedo porque había unas plomaceras, y uno en el medio a salir a esconderse, no, no (...) yo en mi desesperación y el miedo corría a buscar a la niña que estaba en el colegio, eso me tenía asustada (...) Por lo menos ahora hay tranquilidad"(Teresa, 56 años).

Por su parte, una de las mujeres entrevistas dice "Miedo, miedo porque uno se siente incapaz de hacer algo. Para uno la única salida es huir, huir o buscar ayuda, o buscarse en el hogar. Por ejemplo allá la gente riega volantes que dicen que no hay salida después de las 6 de la tarde"(Juana, 24 años). Con respecto a la valoración de respuesta por parte de las autoridades manifestaron que:

"En el putumayo mataron al papá de mi hija (...) ira. Pero yo dejé eso así, ya luego de eso salimos de ahí. Denunciamos pero eso se quedó ahí." (Luz Mary, 36 años). Por otro lado, una de las personas que participaron en el estudio, dice que "Tantas veces fui donde el alcalde y nada (...) pues a mí me da cosa con el gobierno que no cumple lo que problema, y la ayuda eso humanitaria la dan cada 2 años asî" (Teresa, 56 años). En tanto que otra afirma "Nunca han hecho nada, allá no hay autoridad, porque las fuerzas armadas son más en mayoría, ellos se tiene que quedar sin hacer nada porque si no los matan" (Juana, 24 años). 
Finalmente, en cuanto a los retos que se presentan como mujer en el contexto del conflicto armado, una de las participantes afirmó que:

"Contra todo, contra la falta de empleo contra la dominación, el machismo, contra la fuerzas armadas, porque uno como mujer corre mucho peligro. Uno no puede andar por ninguna parte y mucho menos sola. El sitio donde vivimos nosotros lo tienen ellos" (Juana, 24 años)

\section{Discusión}

Con base a lo expresado por las tres participantes, a continuación se expondrá el análisis de los resultados, teniendo en cuenta los postulados teóricos y el objetivo general, el cual pretende conocer si la experiencia del desplazamiento por conflicto armado influye en los comportamientos relacionadas con el rol de género de tres mujeres entre los 20 y 60 años residentes de la ciudad de Cali.

Para comenzar, se resalta el hecho que el desplazamiento como consecuencia del conflicto armado impactó de manera significativa la vida de las tres entrevistadas, ya que percibieron la necesidad de migrar a otro lugar donde pudieran tener mayor seguridad (Comisión Histórica del Conflicto, 2015). De esta manera, se infiere que este acontecimiento las llevó a replantear su manera de estar en el mundo, pues desde sus propias palabras, no encontraron otra opción más que huir, para evitar que los grupos armados al margen de la ley les hicieran daño o decidieran matarlas. Además, el desplazamiento tuvo consecuencias drásticas en su vida, porque significó pérdidas del capital social (Ibañez y Ve- 
lásquez, 2008), como por ejemplo el contacto con los padres, o la muerte del esposo, que tuvieron como resultado una desintegración del contexto familiar, y una reorganización de los roles.

De igual manera, se lograron observar pérdidas de activos, tales como: pérdidas de tierras, de animales y otras pertenencias de las cuales fueron despojadas (Ibañez y Velásquez, 2008). A esto habría que sumarle la dificultad para encontrar un trabajo formal en la ciudad a la que migraron dos de las entrevistadas, puesto que no contaban con experiencia laboral y nivel de escolaridad requerido para ello (Ibañez y Velásquez, 2008). Tal y como se puede observar claramente en lo expresado por Teresa, cuando recuerda que le tocó vender las gallinas que tenía a un precio muy bajo y vender jugos en la plaza del pueblo a donde llegaron, porque no conseguía otro trabajo. Referente a Juana, debió dejar la casa de sus padres y mudarse a la ciudad.

Es así como a lo largo de las entrevistas realizadas, se evidencian memorias cargadas de tristeza y frustración, como lo expresa Juana al decir que ha pasado mucho tiempo sin lograr comunicarse con sus padres, quienes aún viven en territorio con presencia de grupos armados; Luz Mary al evocar que cuando mataron a su pareja sintió mucha rabia y significó un gran reto para ella. Cabe resaltar el gran valor que le atribuyen a la pérdida de capital social que asumieron (Ibañez y $\vee$ elásquez, 2008), lo cual también influyó en el cambio de actividades cotidianas, pues en el caso de Luz Mary, luego de la muerte de su ser querido, decidió cambiar de ciudad de residencia y buscar alternativas de trabajo.

En esta línea de ideas, las actividades cotidianas de Luz Mary y Teresa, se encuentran fuertemente permeadas por los constructos socioculturales del comportamiento femenino, es decir, las la- 
bores relacionadas con lo doméstico, además de las tareas reproductivas y de crianza (Gandulfo, s.f) con las cuales, estas mujeres se identifican. Sin embargo, no ocurre lo mismo con Juana, quien no nombra tener ninguna ni realizar dichas actividades; su postura social se centraba en ser estudiante.

De igual manera, se halló que después del desplazamiento existieron cambios en los roles ejercidos por Luz Mary y Teresa, lo cual va en concordancia con Reynaga (como se cita en Venturoli, 2009), pues se reflejó una reorganización en las funciones que socialmente son concedidas a ellas por el hecho de ser mujeres, debido a que al llegar a la ciudad buscaron trabajos informales para ayudar al sustento económico de su hogar, acciones que son socialmente atribuidas al hombre, donde países como Colombia se ve fuertemente marcada esta distinción, es así como el 61\% de los hogares desplazados son sostenidos por hombres, mientras que el 39\% por la mujer (Ibañez y Velásquez, 2008).

No obstante, aunque se observa que el legado patriarcal sigue vigente, también se rescata la construcción dinámica y permanente de las funciones que estas mujeres realizan y que están influidas por la naturaleza de las exigencias del entorno, así, ellas debieron adaptarse a la situación que estaban experimentando e incluyeron en sus actividades cotidianas, aquellas que anteriormente sólo eran realizadas por sus parejas.

Es así como se concluye que la responsabilidad por la crianza de los hijos, además del sustento económico exige a estas mujeres desarrollar nuevas estrategias y formas de funcionamiento, por lo cual amplían su gama de posibilidades y roles. Se plantean que estas diferencias en los comportamientos socialmente estereotipados para la mujer, pueden tener alguna relación con el momento 
del ciclo de vida en el cual se encuentran las participantes, pues como es sabido, el rol de género es un aspecto socialmente construido (Alcántara, 2013) que puede cambiar generacionalmente, donde históricamente es evidente que las atribuciones alrededor del comportamiento femenino para la mujer eran mucho más marcadas y rígidas, haciendo que Juana tenga una visión de los responsabilidades un poco más independientes de los estereotipos culturalmente construidos para las mujeres.

Finalmente, luego de la revisión realizada en esta investigación, se debe aclarar que los resultados anteriores sólo aplican para las tres mujeres que hicieron parte de la muestra, lo cual sugiere para futuras investigaciones la opción de aumentar la muestra para lograr obtener mayor información. Además, como anotación de las investigadoras, cabe resaltar que después del encuentro con las entrevistados se resalta la importancia de la continuación del acuerdo de paz entre las FARC-EP y el Gobierno colombiano, ya que culminando este paso hacia la terminación del conflicto armado interno, se contribuye a que las historias de migración forzosa y pérdidas relaciones sean cada vez menos.

\section{Referencias}

Alcántara, E. (2013). Identidad sexual/rol de género. Debate feminista, 47, 172-201.

Andrade, J. Alvis, L. Jiménez, L. Redondo, P. y Rodríguez, L. (2017). La vulnerabilidad de la mujer en la guerra y su papel en el posconflicto. El Ágora USB, 17(1), 290-308. 
Bonilla, E. y Rodríguez P. (1997). Recolección de datos cualitativos. En: Más allá de los dilemas de los métodos de investigación. Bogotá: editorial Norma.

Centro de Recursos para el análisis de conflictos (CERAC). (2015). El proceso de Paz con las Farc salva vidas. Recuperado de: http://blog.cerac.org.co/el-proceso-de-paz-con-las-farc-salvavidas

Comisión Histórica del Conflicto (2015). Contribución al entendimiento del conflicto armado en Colombia. Recuperado de: https:// goo.gl/9yhc8v

Gandulfo, M. (s.f). Roles de Género y Desplazamiento Interno en Colombia. Centro Argentino de Estudios Internacionales. Programa América Latina.

Güiza, L. Rodríguez-Barajas, C. Ríos, B.y Moreno, S. (2016). Género y empoderamiento comunitario en un contexto de posconflicto: el caso de Vergara, Cundinamarca (Colombia). Estudios Socio-Jurídicos, 18(2), 117-146.

Ibáñez, A. y Velásquez, A. (2008). El impacto del desplazamiento forzoso en Colombia: condiciones socioeconómicas de la población desplazada, vinculación a los mercados laborales y políticas públicas, Serie Políticas Sociales, 145, 1-74.

La Agencia de la ONU para los Refugiados [ACNUR]. (2015). Tendencias globales, Desplazamiento forzado en 2015, Forzadosa huir. Recuperado de: http://www.acnur.org/fileadmin/scripts/doc. php?file=fileadmin/Documentos/Publicaciones/2016/10627 
Ley número 1448. Congreso Nacional de la República de Colombia, Bogotá D.C, 10 de Junio de 2011.

Ley número 1090 de 2006 (No. 46.383.) Ministerio para la Protección Social de Colombia, 6 de septiembre de 2006.

Miles, M. Tonon, G. y Alvarado, S. (2012). Investigación cualitativa: el análisis temático para el tratamiento de la información desde el enfoque de la fenomenología social. Universitas Humanística 74, 195-225.

Oficina del alto comisionado para la paz (2016). El acuerdo de paz. Bogotá, Colombia, Recuperado de: http://www.acuerdodepaz.gov.co/

Quintana, A. (2006). Metodología de la Investigación Científica Cualitativa. Tópicos de Actualidad. Lima: UNMSM. pp: 56 - 61

Romero-Acosta, K. y Contreras, E. (2015). Revisión teórica sobre el post-conflicto: una oportunidad para empoderar a mujeres víctimas de desplazamiento. CULTURA EDUCACIÓN Y SOCIEDAD, 6(1). Recuperado de: http://revistascientificas.cuc.edu.co/index. php/culturaeducacionysociedad/article/view/757

Salgado, A. (2007). Investigación cualitativa: diseños, evaluación del rigor metodológico y retos. Liberabit, 13(13), 71-78.

Soto, L. (2014). La narración oral como herramienta en la construcción de la memoria colectiva de la violencia. Experiencia con mujeres víctimas de desplazamiento forzado en Colombia. Revista colombiana de ciencias sociales, Fundación Universitaria Luis Amigó, 5(1). 
Torrijos, V. (2015). Cartografía del conflicto: Pautas interpretativas sobre la evolución del conflicto irregular Colombiano.

Unidad de víctimas (2017). Registro Único de Víctimas [RUV]. Recuperado de: http://rni.unidadvictimas.gov.co/RUV

Venturoli, S. (2009). Huir de La Violencia y Construir. Mujeres y desplazamientos por violencia política en Perú. DEP, 11, 46-63. 